\title{
ON THE EXISTENCE OF FUNCTIONS HAVING GIVEN PARTIAL DERIVATIVES ON A CURVE
}

\author{
BY \\ MAURICE SION
}

1. Introduction. Let $A$ be a connected subset of $E_{n}$, where $n$ is an integer greater than 1 , and let $f$ be a function of class $C^{n}$ on $E_{n}$ such that all of its second order partials vanish at every point of $A$, and all of its first order partials vanish at one point of $A$. Then, is $f$ necessarily constant on $A$ ?

This problem $\left({ }^{1}\right)$ was first considered by H. Hopf, while studying the differential geometry of surfaces, and was suggested to the writer by A. P. Morse. It is closely related to the study of the behavior of a function on its critical set.

A critical point of a function $g$ is one at which all the first order partials of $g$ are zero. The set of critical points of $g$ is called the critical set of $g$. In an unpublished paper, M. Morse and A. Sard have shown that, if $g$ is a function of class $C^{m}$ on $E_{n}$ and if $m$ is the greatest integer in $n+(n-3)^{2} / 16$, then $g$ maps its critical set into a set of linear Lebesgue measure zero. A. P. Morse $[1]\left(^{2}\right)$ has proved that this conclusion actually holds whenever $m=n$ for every positive integer $n$. Using this result, A. Sard [2] has been able to generalize this theorem by considering functions on $E_{n}$ to $E_{n^{\prime}}, n$ and $n^{\prime}$ being positive integers. A very slight modification of the proof of Theorem 4.3 in the paper by A. P. Morse enables one to conclude that if $g$ is a function of class $C^{m}$ on $E_{n}$, and if $B$ is such a connected subset of the critical set of $g$ that it has finite Caratheodory $m$-measure whenever $m<n$, then $g$ is constant on $B$. Theorem 2 in the paper by W. M. Whyburn [5] is a special case, taking $m=1$, of this result.

Returning to our original problem, we see, with the help of A. P. Morse's result, that $f$ must be constant on $A$ if we assume that $f$ is of class $C^{n+1}$ instead of class $C^{n}$, or if we let $f$ be of class $C^{n}$ but assume that any two points of $A$ can be joined by such a connected set of zeros of the second order partials of $f$ that it has finite Caratheodory $(n-1)$-measure.

On the other hand, $\mathrm{H}$. Whitney [4] has constructed a set $P$ in $E_{n}$, which is a one-to-one continuous image of the unit interval, and a function $W$ of class $C^{n-1}$ on $E_{n}$ having all its partial derivatives vanish on $P$ and yet assuming the values 0 and 1 respectively at two different points of $P$ One therefore wonders if it is not possible to find a function $f$ of class $C^{n}$ on $E_{n}$ such that on $P$ one of its first order partials coincides with $W$ and all of its other partials

Presented to the Society, September 5, 1953; received by the editors April 30, 1953.

(1) I am indebted to A. Sard for the historical background of this problem.

(2) Numbers in brackets refer to the bibliography at the end. 
are zero. We show that such a function does exist, thus getting a negative solution to the problem stated at the beginning.

In fact, we find sufficient conditions for given functions defined only on some curve to agree on this curve with the partial derivatives of some function defined on the whole space. Repeated applications of this result enable us to see that if $m$ and $n$ are integers, $n \geqq 2$, then there exists a function $f$ of class $C^{n+m-1}$ on $E_{n}$ such that: all of its partials of order greater than $m$ vanish everywhere on $P$; all of its partials vanish at one point of $P$; and yet $f$ is not constant on $P$.

2. Notations and preliminary definitions.

2.1. E $x(\cdots)$ will denote the set of all $x$ such that $(\cdots)$.

2.2. $\{a\}$ will denote the set consisting of the single element $a$.

2.3. $\omega$ will denote the set of all non-negative integers. of $f$.

2.4. $\operatorname{dmn} f$ and $\operatorname{rng} f$ will denote respectively the domain and the range

2.5. $I=\mathrm{E} t(0 \leqq t \leqq 1)$.

2.6. sgmt $x y=\mathrm{E} z(z=(1-t) x+t y$ for some $t$ in $I)$.

2.7. $E_{n}=\mathrm{E} x$ ( $x$ is a sequence of real numbers such that $x_{j}=0$ whenever $n \leqq j \in \omega)$.

2.8. $\|x\|=\left\{\sum_{j \in \omega} x_{j}^{2}\right\}^{1 / 2}$.

2.9. $\mathcal{D}_{j}$ will denote the differential operator such that

$$
\mathcal{D}_{i} f(x)=\lim _{h \rightarrow 0} \frac{f\left(x_{0}, \cdots, x_{j-1}, x_{j}+h, x_{j+1}, \cdots\right)-f(x)}{h} .
$$

2.10. $C^{m}$ will denote the set of all functions having continuous partials through order $m$.

3. Functions with given partial derivatives on a curve. Throughout $\S \S 3$ and 4 we assume that $n$ is a non-negative integer.

Suppose $\psi$ is a homeomorphism on $I$ to a subset of $E_{n+1}$ and that we are given $n+1$ continuous functions $f_{0}, f_{1}, \cdots, f_{n}$ defined on the curve $\psi(I)$. It is not true in general that we can find a function $g$ of class $C^{1}$ on $E_{n+1}$ such that on $\psi(I)$ its $j$ th partial derivative $\mathcal{D}_{j} g$ coincides with $f_{j}, j=0,1, \cdots, n$. Some condition on the curve is necessary.

Now, if, in addition to the above $f_{j}$, we are given continuous functions $f_{s, k}(s, k=0, \cdots, n)$ with domain $\psi(I)$ and wish to find a function $g$ of class $C^{2}$ on $E_{n+1}$ such that for every $x$ in $\psi(I)$

$$
\mathcal{D}_{j} g(x)=f_{j}(x) \text { and } \mathcal{D}_{s} \mathcal{D}_{k} g(x)=f_{s, k}(x)
$$$$
(j, s, k=0, \cdots, n)
$$

we see that we have to impose conditions not only on the curve but also on the given functions. For one thing, we must have $f_{s, k}=f_{k, s} ;$ moreover, the "first order partials" $f_{j}$ and the "second order partials" $f_{s, k}$ must be related by Taylor's formula. Assuming then that the given functions have such prop- 
erties, the condition imposed on the curve in this case need no longer be as stringent as in the previous one.

In general, if suitable "partials" up to order $m$ are prescribed on $\psi(I)$, in order to find a function of class $C^{m}$ on $E_{n+1}$ having the given partials on the curve, the condition imposed on the latter depends on $m$ in such a way that as $m$ increases more curves satisfy it. As we indicate in $\S 8$, for any $m \geqq 2$ there are curves satisfying the condition and yet possessing infinite Caratheodory $(m-1)$-measure. The dependence of the condition on $m$ may be explained heuristically as follows: the "nicer" the given functions are, the "worse" the curve is allowed to be; thus, as $m$ increases, the conditions on the functions become more stringent and hence those on the curve may be relaxed.

We now proceed to give a precise formulation of the theory.

3.1. Conditions on the given functions. We first make some preliminary definitions and introduce a more convenient notation for partial derivatives.

3.1.0. $\sigma k=\sum_{j \in \omega} k_{j}$.

3.1.1. $O_{m}=\mathrm{E} k\left(k \in E_{n+1}, k_{j} \in \omega\right.$ for every $j \in \omega$, and $\left.1 \leqq \sigma k \leqq m\right)$.

3.1.2. $\mathrm{D}_{k} f=\mathscr{D}_{0}^{k_{0}} \cdots \mathscr{D}_{n}^{k_{n}}$.

Thus, if $k$ is a finite sequence of integers, then $\mathrm{D}_{k} f$ is a partial derivative of $f$ of order $\sigma k$, and as $k$ runs over $O_{m}$ we get all the partials of $f$ through order $m$.

3.1.3. We shall say that $R$ is a remainder of order $p$ if and only if $R$ is a function with domain included in $E_{n+1} \times E_{n+1}$ and for each $\epsilon>0$ there is a $\delta>0$ such that: if $(x, y)$ is in $\operatorname{dmn} R$ and $\|x-y\|<\delta$, then $|R(x, y)|<\epsilon$ $\cdot\|x-y\|^{p}$.

The conditions to be imposed on the prescribed "partials" are described by the following definition.

3.1.4. [ $m, F, R, B]$ will stand for the following: $m$ is a positive integer; $F$ and $R$ are functions on $O_{m}$ such that, for every $k$ in $O_{m}, F_{k}$ is a function on $B$ to the reals, $R_{k}$ is a remainder of order $(m-\sigma k)$ with domain $B \times B$, and for every $x$ and $y$ in $B$ :

$$
F_{k}(y)=F_{k}(x)+\sum_{j \in o_{m-\sigma k}} \frac{F_{k+j}(x) \cdot\left(y_{0}-x_{0}\right)^{j_{0}} \cdots\left(y_{n}-x_{n}\right)^{j_{n}}}{j_{0} ! \cdots j_{n} !}+R_{k}(x, y) .
$$

Notice that the $F_{k}$ must be continuous on $B$.

3.2. Conditions on the curve.

3.2.0. $S$ is $a$ subdivision of $[a, b]$ if and only if $a \leqq b$ and, for some positive integer $r, S$ is a function on $\mathrm{E} s(s \in \omega$ and $s \leqq r)$ and

$$
a=S(0)<S(1)<\cdots<S(r)=b .
$$

3.2.1. $D$ is a mesh of $[a, b]$ if and only if $D$ is a sequence of subdivisions of $[a, b]$ such that, for every $i \in \omega$, rng $D_{i} \subset$ rng $D_{i+1}$ and

$$
\lim _{i} \sup _{1 \leqq s \in \operatorname{dmn} D_{i}}\left\{D_{i}(s)-D_{i}(s-1)\right\}=0
$$


3.2.2. If $D$ is a sequence of subdivisions of some interval, we set

$$
D_{i}^{*}(s)=\mathrm{E} r\left[D_{i}(s-1)<D_{i+1}(r-1)<D_{i+1}(r) \leqq D_{i}(s)\right] .
$$

3.2.3. If $S$ is a subdivision of $I$ and $0 \leqq a \leqq b \leqq 1$, we define $S(S ; a, b)$ to be the set of sequences $D$ of subdivisions of $[a, b]$ such that:

$.1 \mathrm{rng} D_{0}=\{a\} \cup\{b\}$;

.2 rng $D_{i} \subset$ rng $D_{i+1} \subset$ rng $S \cup\{a\} \cup\{b\}$ for all. $i \in \omega$;

.3 rng $D_{N}=\{a\} \cup\{b\} \cup$ (rng $S \cap[a, b]$ ) for some $N \in \omega$;

.4 if $1 \leqq s \in \operatorname{dmn} D_{i}$ and $D_{i+1}(r-1)=D_{i}(s-1)$, then $D_{i+1}^{*}(r)=0$ for all $i \in \omega$ and $1 \leqq r \in \mathrm{dmn} D_{i+1}$.

Condition .4 is imposed only to simplify some proofs in $\$ 4$. It could be omitted without affecting the next definitions or any of the results in this paper.

Observe that condition .3 assures us that $D_{N+i}=D_{N}$ and that $\delta(S ; a, b)$ is a finite set.

3.2.4. For a motivation of this definition we refer the reader to Lemma 3.3.13.

If $m \in \omega, \psi$ is a function on $I$ to $E_{n+1}, S$ is a subdivision of $I$, and $0 \leqq a \leqq b$ $\leqq 1$, we define:

$$
\begin{aligned}
& L_{m}(\psi, S ; a, b)=\inf _{D \in \mathcal{S}(S ; a, b)} \sum_{i \in \omega} \sum_{1 \in \in_{s} \leq \mathrm{dmn} D_{i}} \sum_{r \in D_{i}^{*}(s)} \\
& \quad \sum_{p=1}^{m}\left\{\left\|\psi\left(D_{i}(s-1)\right)-\psi\left(D_{i+1}(r-1)\right)\right\|\left\|^{m-p} .\right\| \psi\left(D_{i+1}(r-1)\right)-\psi\left(D_{i+1}(r)\right) \|^{p}\right\} .
\end{aligned}
$$

The conditions to be imposed on the curve are described by the next two definitions, and a motivation for them is to be found in Lemmas 3.3.14 and 3.3.15.

3.2.5. $\langle m, \psi, T\rangle$ will stand for the following: $m$ is a positive integer; $\psi$ is a homeomorphism on $I$ to a subset of $E_{n+1} ; T$ is a mesh of $I$; for every $t$ in $I$ there exists a $K<\infty$ such that, if $\alpha_{i}(t)=\sup \mathrm{E} s\left(T_{i}(s) \leqq t\right)$, then

$$
.1 \quad \sum_{s=1}^{\alpha_{i}(t)} L_{m}\left(\psi, T_{i+p} ; T_{i}(s-1), T_{i}(s)\right)+L_{m}\left(\psi, T_{i+p} ; T_{i}\left(\alpha_{i}(t)\right), t\right)<K
$$

for every $i$ and $p$ in $\omega$.

3.2.6. $\langle\langle m, \psi, T\rangle\rangle$ will stand for the following: $m$ is a positive integer; $\psi$ is a homeomorphism on $I$ to a subset of $E_{n+1} ; T$ is a mesh of $I$; there exists a $K<\infty$ such that, for every $i \in \omega$ and $0 \leqq a \leqq b \leqq 1$ :

$$
L_{m}\left(\psi, T_{i} ; a, b\right)<K\|\psi(b)-\psi(a)\|^{m} .
$$

3.3. Construction of a function having given partial derivatives on a curve. Throughout $\S 3.3$ we assume that $\psi$ is a homeomorphism on $I$ to a subset of $E_{n+1}, T$ is a mesh to $I$, and $[m, F, R, \psi(I)]$. 
We wish to show that there exists a function $\bar{g}$ of class $C^{m}$ on $E_{n+1}$ such that for every $x$ in $\psi(I)$ and $k$ in $O_{m}$ we have $\mathrm{D}_{k} \bar{g}(x)=F_{k}(x)$. In order to prove the existence of such a function $\bar{g}$, it suffices to construct a function $g$ on $\psi(I)$ to the reals such that, for every $x$ and $y$ on the curve, we have:

$$
g(y)=g(x)+\sum_{k \in o_{m}} F_{k}(x) \frac{\left(y_{0}-x_{0}\right)^{k_{0}} \cdots\left(y_{n}-x_{n}\right)^{k_{n}}}{k_{0} ! \cdots k_{n} !}+R_{0}(x, y)
$$

where $R_{0}$ is a remainder of order $m$ with domain $\psi(I) \times \psi(I)$. For then, we only have to apply the well known result of Whitney's [3] to extend the functions to the whole space and get $\bar{g}$.

Before defining $g$ we introduce the following notations and abbreviations.

$$
A_{i}\left(t, t^{\prime}\right)=\mathrm{E} s\left(t \leqq T_{i}(s-1)<T_{i}(s) \leqq t^{\prime}\right) ;
$$

$$
t_{i}^{*}=\sup \left\{\operatorname{rng} T_{i} \cap[0, t]\right\}
$$

$$
h(x, y)=\sum_{k \in o_{m}} F_{k}(x) \frac{\left(y_{0}-x_{0}\right)^{k_{0}} \cdots\left(y_{n}-x_{n}\right)^{k_{n}}}{k_{0} ! \cdots k_{n} !} ;
$$

3.3. $\quad R(x, y, z)=\sum_{k \in o_{m}} R_{k}(x, y) \frac{\left(z_{0}-y_{0}\right)^{k_{0}} \cdots\left(z_{n}-y_{n}\right)^{k_{n}}}{k_{0} ! \cdots k_{n} !}$;

3.3.4. $\quad M=\sum_{k \in o_{m}} \frac{1}{k_{0} ! \cdots k_{n} !}$.

Next, we define $f$ to be the sequence of functions on $I$ such that for every $i \in \omega$ and every $t$ in $I$

$$
f_{i}(t)=\sum_{s \in A_{i}(0, t)} h\left(\psi\left(T_{i}(s-1)\right), \psi\left(T_{i}(s)\right)\right)+h\left(\psi\left(t_{i}^{*}\right), \psi(t)\right) .
$$

Finally, we define the functions $g^{\prime}, g, R_{0}$ by

$$
g^{\prime}(t)=\lim _{i} f_{i}(t)
$$

for every $t$ in $I$;

$$
g(x)=g^{\prime}\left(\psi^{-1}(x)\right)
$$

for every $x$ in $\psi(I)$;

$$
R_{0}(x, y)=g(y)-g(x)-h(x, y)
$$
for every $x$ and $y$ in $\psi(I)$.

The rest of this section is devoted to proving that $g(x)$ is a finite real number for every $x$ in $\psi(I)$ if $\langle m, \psi, T\rangle$, and that $R_{0}$ is a remainder of order $m$ if, in addition, we assume $\langle\langle m, \psi, T\rangle\rangle$.

3.3.9. Lemma. If $x, y, z$ are in $\psi(I)$, then

$$
h(x, y)+h(y, z)-h(x, z)=R(x, y, z) .
$$

Proof. Let $h^{\prime}$ be the function such that, for any $w$ in $E_{n+1}, h^{\prime}(w)=h(x, w)$. Then, $h^{\prime}$ is a polynomial of degree $m$, and for any $k$ in $O_{m}$ we have: 


$$
\mathrm{D}_{k} h^{\prime}(w)=\sum_{p \in O_{m}, p \geqq k} F_{p}(x) \frac{\left(w_{0}-x_{0}\right)^{p_{0}-k_{0}} \cdots\left(w_{n}-x_{n}\right)^{p_{n}-k_{n}}}{\left(p_{0}-k_{0}\right) ! \cdots\left(p_{n}-k_{n}\right) !} .
$$

(For $p$ and $k$ in $O_{m}, p \geqq k$ means: $p_{i} \geqq k_{i}$ for all $i$.) Hence, by Taylor's formula we see that

$$
\begin{aligned}
& h(x, z)=h(x, y)+\sum_{k \in o_{m}} \mathrm{D}_{k} h^{\prime}(y) \frac{\left(z_{0}-y_{0}\right)^{k_{0}} \cdots\left(z_{n}-y_{n}\right)^{k_{n}}}{k_{0} ! \cdots k_{n} !} \\
& =h(x, y)+\sum_{k \in o_{m}}\left\{\left\{\sum_{p \in o_{m}, p \geqq h} F_{p}(x) \frac{\left(y_{0}-x_{0}\right)^{p_{0}-k_{0}} \cdots\left(y_{n}-x_{n}\right)^{p_{n}-k_{n}}}{\left(p_{0}-k_{0}\right) ! \cdots\left(p_{n}-k_{n}\right) !}\right\}\right. \\
& \left.\frac{\left(z_{0}-y_{0}\right)^{k_{0}} \cdots\left(z_{n}-y_{n}\right)^{k_{n}}}{k_{0} ! \cdots k_{n} !}\right\} \text {. }
\end{aligned}
$$

On the other hand, from the definition 3.3.2 and the equation in 3.1.4 we see that

$$
\begin{aligned}
& h(y, z)=\sum_{k \in o_{m}}\left\{\left\{F_{k}(x)+\sum_{j \in o_{m-\sigma k}} F_{k+j}(x) \frac{\left(y_{0}-x_{0}\right)^{j_{0}} \cdots\left(y_{n}-x_{n}\right)^{j_{n}}}{j_{0} ! \cdots j_{n} !}\right.\right. \\
& \left.\left.+R_{k}(x, y)\right\} \frac{\left(z_{0}-y_{0}\right)^{k_{0}} \cdots\left(z_{n}-y_{n}\right)^{k_{n}}}{k_{0} ! \cdots k_{n} !}\right\} \\
& =[3.3 .3] \sum_{k \in o_{m}}\left\{\left\{\sum_{p \in O_{m}, p \geqq k} F_{p}(x) \frac{\left(y_{0}-x_{0}\right)^{p_{0}-k_{0}} \cdots\left(y_{n}-x_{n}\right)^{p_{n}-k_{n}}}{\left(p_{0}-k_{0}\right) ! \cdots\left(p_{n}-k_{n}\right) !}\right\}\right. \\
& \left.\frac{\left(z_{0}-y_{0}\right)^{k_{0}} \cdots\left(z_{n}-y_{n}\right)^{k_{n}}}{k_{0} ! \cdots k_{n} !}\right\}+R(x, y, z) \\
& =h(x, z)-h(x, y)+R(x, y, z),
\end{aligned}
$$

and the desired conclusion is at hand.

3.3.10. Lemma. If $0 \leqq a \leqq S_{0}<S_{1}<\cdots<S_{r}=b \leqq 1$, then

$$
\sum_{i=1}^{r} h\left(\psi\left(S_{i-1}\right), \psi\left(S_{i}\right)\right)-h(\psi(a), \psi(b))=\sum_{i=1}^{r} R\left(\psi(a), \psi\left(S_{i-1}\right), \psi\left(S_{i}\right)\right) .
$$

Proof. $\sum_{i=1}^{r} h\left(\psi\left(S_{i-1}\right), \psi\left(S_{i}\right)\right)-h(\psi(a), \psi(b))$

$$
\begin{aligned}
& =\sum_{i=1}^{r}\left\{h\left(\psi(a), \psi\left(S_{i-1}\right)\right)+h\left(\psi\left(S_{i-1}\right), \psi\left(S_{i}\right)\right)-h\left(\psi(a), \psi\left(S_{i}\right)\right)\right\} \\
& =[3.3 .9] \sum_{i=1}^{r} R\left(\psi(a), \psi\left(S_{i-1}\right), \psi\left(S_{i}\right)\right) .
\end{aligned}
$$


3.3.11. Lemma. If $0 \leqq a \leqq b \leqq 1, S$ is a subdivision of $[a, b], D$ is in $\mathcal{S}(S ; a, b)$, then

$$
\begin{array}{rl}
\sum_{1 \leqq j} \in \operatorname{dmn} S & h(\psi(S(j-1)), \psi(S(j)))-h(\psi(a), \psi(b)) \\
= & \sum_{i \in \omega} \sum_{1 \leqq j \in \operatorname{dmn} D_{i} r \in D_{i}^{*}(j)} R\left(\psi\left(D_{i}(j-1)\right), \psi\left(D_{i+1}(r-1)\right), \psi\left(D_{i+1}(r)\right)\right) .
\end{array}
$$

Proof. Let $r_{i}^{j}=\inf \operatorname{E} r\left(D_{i}(j-1)<D_{i+1}(r)\right)$. Notice that, if $1 \leqq j \in \operatorname{dmn} D_{i}$, then $D_{i}(j-1)=D_{i+1}\left(r_{i}^{j}-1\right)$, and that $1 \leqq r \in \operatorname{dmn} D_{i+1}$ if and only if, for some $1 \leqq j \in \operatorname{dmn} D_{i}, r \in D_{i}^{*}(j)$ or $r=r_{i}^{j}-1$. Referring to 3.2.3, let $N$ be an integer such that $S=D_{N}$ and observe that:

$$
\begin{aligned}
& \sum_{1 \leqq} j_{\operatorname{dmn}} S(\psi(S(j-1)), \psi(S(j)))-h((a),(b)) \\
= & \sum_{i=0}^{N-1}\left\{\sum_{1 \leqq r \in \operatorname{dmn} D_{i+1}} h\left(\psi\left(D_{i+1}(r-1)\right), \psi\left(D_{i+1}(r)\right)\right)\right. \\
& \left.-\sum_{1 \leqq j \in \operatorname{dmn} D_{i}} h\left(\psi\left(D_{i}(j-1)\right), \psi\left(D_{i}(j)\right)\right)\right\} \\
= & \sum_{i=0}^{N-1}\left\{\sum _ { 1 \leqq j \in \operatorname { d m n } D _ { i } } \left\{h\left(\psi\left(D_{i}(j-1)\right), \psi\left(D_{i+1}\left(r_{i}^{j}\right)\right)\right)\right.\right. \\
& \left.\left.+\sum_{r \in D_{i}^{*}(j)} h\left(\psi\left(D_{i+1}(r-1)\right), \psi\left(D_{i+1}(r)\right)\right)-h\left(\psi\left(D_{i}(j-1)\right), \psi\left(D_{i}(j)\right)\right)\right\}\right\} \\
= & {[3.3 .10] \sum_{i=0}^{N-1} \sum_{1 \leqq j} \sum_{\operatorname{dmn} D_{i}} R\left(\psi\left(D_{i}(j-1)\right), \psi\left(D_{i+1}^{*}(r)\right.\right.}
\end{aligned}
$$

Since $D_{N+i}^{*}(j)=0$, we get no more terms if we take the first summation to be over $\omega$.

3.3.12. Lemma. If, for every $k$ in $O_{m},\left|R_{k}(x, y)\right|<\epsilon\|y-x\|^{m-\sigma k}$, then

$$
|R(x, y, z)|<\epsilon M \sum_{p=1}^{m}\|y-x\|\left\|^{m-p}\right\| z-y \|^{p} \text {. }
$$

Proof.

$$
\begin{aligned}
& |R(x, y, z)| \leqq \sum_{k \in o_{m}}\left|R_{k}(x, y)\right| \frac{\left|z_{0}-y_{0}\right|^{k_{0}} \cdots\left|z_{n}-y_{n}\right|^{k_{n}}}{k_{0} ! \cdots k_{n} !} \\
& \leqq \sum_{p=1}^{m} \sum_{\sigma k=p} \frac{\epsilon\|y-x\|\left\|^{m-\sigma k}\right\|_{z}-y \|^{\sigma o}}{k_{0} ! \cdots k_{n} !} \\
& \leqq \in M \sum_{p=1}^{m}\|y-x\|^{m-p}\|z-y\|^{p} \text {. }
\end{aligned}
$$


3.3.13. Lemma. If $0 \leqq a \leqq b \leqq 1, S$ is a subdivision of $[a, b]$,

$$
\left|R_{k}\left(\psi(t), \psi\left(t^{\prime}\right)\right)\right| \leqq \epsilon\left\|\psi(t)-\psi\left(t^{\prime}\right)\right\|^{m-\sigma b}
$$

for every $k$ in $O_{m}$ and every $t$ and $t^{\prime}$ in $[a, b]$, then

$$
\left|\sum_{1 \leqq j \in \mathrm{dmn} S} h(\psi(S(j-1)), \psi(S(j)))-h(\psi(a), \psi(b))\right| \leqq \epsilon M L_{m}(\psi, S ; a, b) .
$$

Proof. Apply 3.3.11, 3.3.12 and compare with definition 3.2.4.

3.3.14. TheOREM. If $\langle m, \psi, T\rangle$, then for every $x$ in $\psi(I), g(x)$ is a finite real number.

Proof. Given $t$ in $I, \epsilon>0$, we show that there is an integer $i$ such that, for every $j$ in $\omega,\left|f_{i+j}(t)-f_{i}(t)\right| \leqq \epsilon$.

Let $K$ satisfy equation 3.2.5.1 and $\epsilon^{\prime}=\epsilon / M K$. Pick $\delta$ so that $0<\delta<1$, and whenever $x, y$ are in $\psi(I)$ and $\|y-x\| \leqq \delta$, then $\left|R_{k}(x, y)\right| \leqq \epsilon^{\prime}\|y-x\|^{m-\sigma k}$ for every $k$ in $O_{m}$. Next choose $i$ so that, if $T_{i}(s-1) \leqq a \leqq b \leqq T_{i}(s)$ for any $1 \leqq s$ $\in \operatorname{dmn} T_{i}$, then $\|\psi(b)-\psi(a)\| \leqq \delta$. For any $j$ in $\omega$ we have:

$$
\begin{aligned}
\mid f_{i+j}(t) & -f_{i}(t) \mid \\
= & \mid \sum_{s \in A_{i+j}(0, t)} h\left(\psi\left(T_{i+j}(s-1)\right), \psi\left(T_{i+j}(s)\right)\right)+h\left(\psi\left(t_{i+j}^{*}\right), \psi(t)\right) \\
& -\sum_{s \in A_{i}(0, t)} h\left(\psi\left(T_{i}(s-1)\right), \psi\left(T_{i}(s)\right)\right)-h\left(\psi\left(t_{i}^{*}\right), \psi(t)\right) \mid \\
= & \mid \sum_{s \in A_{i}(0, t)}\left\{\sum_{r \in A_{i+j}\left(T_{\left.i(s-1), T_{i}(s)\right)}\right.} h\left(\psi\left(T_{i+j}(r-1)\right), \psi\left(T_{i+j}(r)\right)\right)\right. \\
- & \left.h\left(\psi\left(T_{i}(s-1)\right), \psi\left(T_{i}(s)\right)\right)\right\} \\
& +\sum_{s \in A_{i+j}\left(t_{i}^{*}, t\right)} h\left(\psi\left(T_{i+j}(s-1)\right), \psi\left(T_{i+j}(s)\right)\right) \\
& +h\left(\psi\left(t_{i+j}^{*}\right), \psi(t)\right)-h\left(\psi\left(t_{i}^{*}\right), \psi(t)\right) \mid \\
\leqq & {[3.3 .13] \epsilon_{\epsilon^{\prime} M .} \sum_{s \in A_{i}(0, t)} L_{m}\left(\psi, T_{i+j} ; T_{i}(s-1), T_{i}(s)\right) } \\
& +\epsilon^{\prime} M L_{m}\left(\psi, T_{i+j} ; t_{i}^{*}, t\right) \leqq \epsilon .
\end{aligned}
$$

3.3.15. Lemma. If $\langle\langle m, \psi, T\rangle\rangle$, then for any $\epsilon>0$, there is $a \delta>0$ such that if $t$ is in $\operatorname{rng} T_{i}$ for any $i$ in $\omega$ and $t<t^{\prime} \leqq 1$ and $\left\|\psi\left(t^{\prime}\right)-\psi(t)\right\| \leqq \delta$, then

$$
\left|f_{i+j}\left(t^{\prime}\right)-f_{i+j}(t)-h\left(\psi(t), \psi\left(t^{\prime}\right)\right)\right| \leqq \epsilon\left\|\psi\left(t^{\prime}\right)-\psi(t)\right\| m
$$

for every $j \in \omega$. 
Proof. Let $K$ satisfy equation 3.2.6.1. Next choose $\delta^{\prime}>0$ so that, if $x$ and $y$ are in $\psi(I)$ and $\|x-y\| \leqq \delta^{\prime}$, then $\left|R_{k}(x, y)\right| \leqq(\epsilon / M K)\|y-x\|^{m-\sigma k}$ for every $k$ in $O_{m}$. Finally, choose $\delta>0$ so that, if $0 \leqq a \leqq b \leqq 1$ and $\|\psi(b)-\psi(a)\| \leqq \delta$, then diameter $\psi[a, b] \leqq \delta^{\prime}$. Then, if $i \in \omega, t \in \operatorname{rng} T_{i}, t \leqq t^{\prime} \leqq 1$, and $\| \psi\left(t^{\prime}\right)$ $-\psi(t) \|<\delta$, we have:

$$
\begin{aligned}
& \left|f_{i+j}\left(t^{\prime}\right)-f_{i+j}(t)-h\left(\psi(t), \psi\left(t^{\prime}\right)\right)\right| \\
& =\left|\sum_{s \in A_{i+j}\left(t, t^{\prime}\right)} h\left(\psi\left(T_{i+j}(s-1)\right),\left(T_{i+j}(s)\right)\right)+h\left(\psi\left(t_{i+j}^{*}\right), \psi\left(t^{\prime}\right)\right)-h\left(\psi(t), \psi\left(t^{\prime}\right)\right)\right| \\
& \leqq[3.3 .13] \frac{\epsilon}{M K} M L_{m}\left(\psi, T_{i+j} ; t, t^{\prime}\right) \leqq \epsilon\left\|\psi\left(t^{\prime}\right)-\psi(t)\right\| \|^{m} .
\end{aligned}
$$

3.3.16. Theorem. If $\langle m, \psi, T\rangle$ and $\langle\langle m, \psi, T\rangle\rangle$, then for any $\epsilon>0$ there is $a \quad \delta>0$ such that, if $0 \leqq t \leqq t^{\prime} \leqq 1$ and $\left\|\psi\left(t^{\prime}\right)-\psi(t)\right\| \leqq \delta$, then $\mid g^{\prime}\left(t^{\prime}\right)-g^{\prime}(t)$ $-h\left(\psi(t), \psi\left(t^{\prime}\right)\right) \mid \leqq \epsilon\left\|\psi\left(t^{\prime}\right)-\psi(t)\right\|^{m}$.

Proof. Let $\epsilon^{\prime}=\epsilon /(M+1)$ where $M$ satisfies equation 3.3.4. Choose $\delta>0$ to satisfy 3.3 .15 with " $\epsilon$ " replaced by " $\epsilon$ " Then pick $i \in \omega$ so that, if $T_{i}(s-1)$ $\leqq a \leqq b \leqq T_{i}(s)$ for any $1 \leqq s \in \mathrm{dmn} T_{i}$, then

$$
\|\psi(b)-\psi(a)\| \leqq \delta .
$$

Hence, for any integer $j \geqq i, 0 \leqq t \leqq t^{\prime} \leqq 1,\left\|\psi\left(t^{\prime}\right)-\psi(t)\right\| \leqq \delta$ :

$$
\begin{aligned}
&\left|f_{j}\left(t^{\prime}\right)-f_{j}(t)-h\left(\psi(t), \psi\left(t^{\prime}\right)\right)\right| \\
& \leqq\left|f_{j}\left(t_{j}^{*}\right)+h\left(\psi\left(t_{j}^{*}\right), \psi(t)\right)-f_{j}(t)\right|+\left|f_{j}\left(t^{\prime}\right)-f_{j}\left(t_{j}^{*}\right)-h\left(\psi\left(t_{j}^{*}\right), \psi\left(t^{\prime}\right)\right)\right| \\
& \quad+\left|h\left(\psi\left(t_{j}^{*}\right), \psi\left(t^{\prime}\right)\right)-h\left(\psi\left(t_{j}^{*}\right), \psi(t)\right)-h\left(\psi(t), \psi\left(t^{\prime}\right)\right)\right| \\
& \leqq {[3.3 .5,3.3 .15,3.3 .9] 0+\epsilon^{\prime}\left\|\psi\left(t^{\prime}\right)-\psi\left(t_{j}^{*}\right)\right\|{ }^{m} } \\
& \quad+\left|R\left(\psi\left(t_{j}^{*}\right), \psi(t), \psi\left(t^{\prime}\right)\right)\right| \\
& \leqq {[3.3 .12] \epsilon^{\prime}\left\|\psi\left(t^{\prime}\right)-\psi\left(t_{j}^{*}\right)\right\|{ }^{m}+\epsilon^{\prime} M \sum_{p=1}^{m}\left\|\psi(t)-\psi\left(t_{j}^{*}\right)\right\|^{m-p}\left\|\psi(t)-\psi\left(t^{\prime}\right)\right\|^{p} }
\end{aligned}
$$

Letting $j \rightarrow \infty$, since $\psi\left(t_{j}^{*}\right) \rightarrow \psi(t)$, we have:

$\left|g^{\prime}\left(t^{\prime}\right)-g^{\prime}(t)-h\left(\psi(t), \psi\left(t^{\prime}\right)\right)\right| \leqq \epsilon^{\prime}(M+1)\left\|\psi\left(t^{\prime}\right)-\psi(t)\right\|\left\|^{m}=\epsilon\right\| \psi\left(t^{\prime}\right)-\psi(t) \|^{m}$.

3.3.17. TheоReм. If $\langle m, \psi, T\rangle$ and $\langle\langle m, \psi, T\rangle\rangle$, then $R_{0}$ is a remainder of order $m$.

Proof. Given $\epsilon>0$, choose $\delta$ as given by 3.3 .16 with " $\epsilon$ " replaced by " $\epsilon / 2 m M$." Let $x$ and $y$ be in $\psi(I)$ and $\|x-y\| \leqq \delta$. Suppose $\psi^{-1}(x) \leqq \psi^{-1}(y)$. Then, by 3.3.16, we have:

$\left|R_{0}(x, y)\right|=|g(y)-g(x)-h(x, y)| \leqq(\epsilon / 2 m M)\|y-x\|^{m} \leqq(\epsilon / 2)\|y-x\|^{m}$.

Now, by 3.3.9: 


$$
\begin{aligned}
|h(x, y)+h(y, x)| & =|h(x, y)+h(y, x)-h(x, x)|=|R(x, y, x)| \\
& \leqq[3.3 .12](\epsilon / 2 m M) M \sum_{p=1}^{m}\|y-x\|^{m} \\
& =(\epsilon / 2 m M) M m\|y-x\|^{m}=(\epsilon / 2)\|y-x\|^{m} .
\end{aligned}
$$

Hence:

$$
\begin{aligned}
\left|R_{0}(y, x)\right| & =|g(x)-g(y)-h(y, x)| \\
& \leqq|g(x)-g(y)+h(x, y)|+|h(y, x)+h(x, y)| \leqq \epsilon\|y-x\|^{m} .
\end{aligned}
$$

3.3.18. TheOREM. Let $A$ be a closed subset of $E_{n+1}$. For each $k$ in $O_{m} \cup\{0\}$ let $r_{k}$ be a remainder of order $(m-\sigma k)$ with domain $(A \times A)$, and $G_{k}$ be such $a$ function on $A$ that if $x$ and $y$ are in $A$, then:

$$
G_{k}(y)=G_{k}(x)+\sum_{j \in o_{m-\sigma k}} G_{k+j}(x) \frac{\left(y_{0}-x_{0}\right)^{j_{0}} \cdots\left(y_{n}-x_{n}\right)^{j_{n}}}{j_{0} ! \cdots j_{n} !}+r_{k}(x, y) .
$$

Then, there is a function $\bar{G}$ on $E_{n+1}$ such that $\bar{G}$ is of class $C^{m}$, and, for any $x$ in $A$ and $k$ in $O_{m} \cup\{0\}, \mathrm{D}_{k} \bar{G}(x)=G_{k}(x)$.

Proof. See H. Whitney [3].

3.3.19. ThEorem. If $\langle m, \psi, T\rangle,\langle\langle m, \psi, T\rangle\rangle$, and $[m, F, R, \psi(I)]$, then there exists a function $\bar{g}$ on $E_{n+1}$ such that $\bar{g}$ is of class $C^{m}$ and, for any $k$ in $O_{m}$ and $x$ in $\psi(I), \mathrm{D}_{k} \bar{g}(x)=F_{k}(x)$.

Proof. Apply Theorem 3.3.18 by taking $A=\psi(I), G_{0}=g, r_{0}=R_{0}$, and for $k$ in $O_{m}, G_{k}=F_{k}, r_{k}=R_{k}$.

3.3.20. Remark. As we show in $4.7,\langle\langle m, \psi, T\rangle\rangle$ implies $\langle m, \psi, T\rangle$. Thus, in 3.3.19, we need only assume $\langle\langle m, \psi, T\rangle\rangle$.

4. Consequences of $\langle\langle m, \psi, T\rangle\rangle$.

4.1. Lemma. If $r \in \omega ; x_{0}, x_{1}, \cdots, x_{r}$ are points in $E_{n+1} ; m \geqq 1 ; K \geqq 0$; $\left\|x_{0}-x_{r}\right\| \leqq K$ and

$$
\sum_{i=1}^{r-1}\left\|x_{0}-x_{i}\right\|{ }^{m-1}\left\|x_{i}-x_{i+1}\right\| \leqq K^{m} ;
$$

then $\left\|x_{0}-x_{i}\right\| \leqq 2 K$, for $i=1, \cdots, r-1$.

Proof. Let $s$ be the smallest integer for which

$$
\inf _{s \leqq i<r}\left\|x_{0}-x_{i}\right\| \mathbf{l}>K \text {. }
$$

Then

$$
K^{m-1} \sum_{i=s}^{r-1}\left\|x_{i}-x_{i+1}\right\|<\sum_{i=s}^{r-1}\left\|x_{0}-x_{i}\right\| m-1\left\|x_{i}-x_{i+1}\right\| \leqq K^{m}
$$


Hence $\sum_{i=s}^{r-1}\left\|x_{i}-x_{i+1}\right\|<K$ and for $k=s, s+1, \cdots, r-1$, we have

$$
\left\|x_{0}-x_{k}\right\| \leqq\left\|x_{0}-x_{r}\right\|+\sum_{i=k}^{r-1}\left\|x_{i}-x_{i+1}\right\|<2 K .
$$

If $s>1$, then, from the choice of $s,\left\|x_{0}-x_{s-1}\right\| \leqq K$. If $s>2$, we can repeat the above argument replacing " $r$ " by " $s-1$." By finite induction then we have the desired result.

4.2. Lemma. If $D$ is a sequence of subdivisions of $[a, b]$ such that, for some $i \in \omega$, rng $D_{i} \subset$ rng $D_{i+1}$, and $1 \leqq r \in \operatorname{dmn} D_{i+1}$, then there exists an $s$ in $\operatorname{dmn} D_{i}$ for which $r \in D_{i}^{*}(s)$ if and only if, for every $s^{\prime}$ in $\operatorname{dmn} D_{i}, D_{i+1}(r-1) \neq D_{i}\left(s^{\prime}-1\right)$.

Proof. The necessity follows immediately from Definition 3.2.2. Now, assume that $D_{i+1}(r-1) \neq D_{i}\left(s^{\prime}-1\right)$ for every $s^{\prime}$ in $\operatorname{dmn} D_{i}$. Let $s$ $=\sup \mathrm{E}_{p} \quad\left(D_{i}(p-1)<D_{i+1}(r-1)\right)$. Then $D_{i}(s-1)<D_{i+1}(r-1)<D_{i+1}(r)$ $\leqq D_{i}(s)$ since rng $D_{i} \subset \operatorname{rng} D_{i+1}$.

4.3. Lemma. Let $S$ be a subdivision of $I, D \in \mathcal{S}(S ; a, b), \psi$ a function on $I$ to $E_{n+1}, 1 \leqq m \in \omega, K \geqq 1$, and

$$
\begin{aligned}
\sum_{i \in \omega} \sum_{1 \leqq s \in d m n} \sum_{D_{i}} \sum_{r \in D_{i}^{*}(s)} \sum_{p=1}^{m}\left\{\left\|\psi\left(D_{i}(s-1)\right)-\psi\left(D_{i+1}(r-1)\right)\right\|^{m-p}\right. \\
\left.\cdot\left\|\psi\left(D_{i+1}(r-1)\right)-\psi\left(D_{i+1}(r)\right)\right\|^{p}\right\} \leqq K^{m}\|\psi(b)-\psi(a)\|^{m} .
\end{aligned}
$$

Then $\left\|\psi\left(D_{i}(r-1)\right)-\psi\left(D_{i}(r)\right)\right\| \leqq 2 K\|\psi(b)-\psi(a)\|$ for all $i \in \omega$ and $1 \leqq r$ $\in \operatorname{dmn} D_{i}$.

Proof. If $i=0$ the conclusion is immediate. Also, if $r \in D_{i}^{*}(s)$ for some $s$ in $\operatorname{dmn} D_{i}$, then $\left\|\psi\left(D_{i+1}(r-1)\right)-\psi\left(D_{i+1}(r)\right)\right\| \leqq K\|\psi(b)-\psi(a)\|$. In view of 4.2, the only case left is when $D_{i+1}(r-1)=D_{i}(s-1)$ for some $s$ in $\operatorname{dmn} D_{i}$. We use induction. If $i=0$, the result follows from 4.1. Assume it is true for $i=j$ and let $i=j+1$.

If $s \in D_{j}^{*}\left(s^{\prime}\right)$ for some $1 \leqq s^{\prime} \in \operatorname{dmn} D_{j}$, then

$$
\left\|\psi\left(D_{j+1}(s-1)\right)-\psi\left(D_{j+1}(s)\right)\right\| \leqq K\|\psi(b)-\psi(a)\|
$$

and hence, by 4.1 :

$$
\left\|\psi\left(D_{j+2}(r-1)\right)-\psi\left(D_{j+2}(r)\right)\right\| \leqq 2 K\|\psi(b)-\psi(a)\| .
$$

If $s$ is not in $D_{j}^{*}\left(s^{\prime}\right)$ for any $s^{\prime}$ in $\mathrm{dmn} D_{j}$, then by $4.2, D_{j+1}(s-1)=D_{j}\left(s^{\prime}-1\right)$ for some $s^{\prime}$ in dmn $D_{j}$. Hence, by condition 3.2.3.4, $D_{j+1}^{*}(s)=0$ and therefore $D_{j+2}(r)=D_{j+1}(s)$. Thus, by the induction hypothesis:

$$
\begin{aligned}
\left\|\psi\left(D_{i+2}(r-1)\right)-\psi\left(D_{i+2}(r)\right)\right\| & =\left\|\psi\left(D_{j+1}(s-1)\right)-\psi\left(D_{j+1}(s)\right)\right\| \\
& \leqq 2 K\|\psi(b)-\psi(a)\| .
\end{aligned}
$$


4.4. Theorem. If $\langle\langle m, \psi, T\rangle\rangle$, then $\langle\langle m+1, \psi, T\rangle\rangle$.

Proof. Choose $K \geqq 1$ so as to satisfy 3.2.6.1. Given $0 \leqq a \leqq b \leqq 1$ and $j \in \omega$, choose $D \in \mathcal{S}\left(T_{j} ; a, b\right)$ so that

$$
\begin{aligned}
\sum_{i \in \omega} \sum_{1 \leqq s \in d m n} \sum_{D_{i}} \sum_{r} \sum_{D_{i}^{*}(s)} \sum_{p=1}^{m}\left\{\left\|\psi\left(D_{i}(s-1)\right)-\psi\left(D_{i+1}(r-1)\right)\right\|^{m-p}\right. \\
\left.\cdot\left\|\psi\left(D_{i+1}(r-1)\right)-\psi\left(D_{i+1}(r)\right)\right\|^{p}\right\} \leqq K^{m}\|\psi(b)-\psi(a)\|^{m} .
\end{aligned}
$$

Notice that for all $i \in \omega$, if $r \in D_{i}^{*}(s)$ for some $1 \leqq s \in \operatorname{dmn} D_{i}$, then

$$
\left\|\psi\left(D_{i+1}(r-1)\right)-\psi\left(D_{i+1}(r)\right)\right\| \leqq K\|\psi(b)-\psi(a)\| .
$$

Also, by Lemma 4.1 , if $r \in D_{0}^{*}(1)$, then

$$
\left\|\psi\left(D_{0}(0)\right)-\psi\left(D_{1}(r-1)\right)\right\| \leqq 2 K\|\psi(b)-\psi(a)\| .
$$

And, if $1 \leqq i \in \omega$ and $r \in D_{i}^{*}(s)$ for some $1 \leqq s \in \operatorname{dmn} D_{i}$, then by 3.2.3.4 $D_{i}(s-1) \neq D_{i-1}\left(s^{\prime}-1\right)$ for all $s^{\prime}$ in $\operatorname{dmn} D_{i-1}$, and hence by $4.2, s \in D_{i-1}^{*}\left(s^{\prime}\right)$ for some $s^{\prime}$ in dmn $D_{i-1}$. Thus, $\left\|\psi\left(D_{i}(s-1)\right)-\psi\left(D_{i}(s)\right)\right\| \leqq K\|\psi(b)-\psi(a)\|$, and by 4.1 we have:

$$
\left\|\psi\left(D_{i}(s-1)\right)-\psi\left(D_{i+1}(r-1)\right)\right\| \leqq 2 K\|\psi(b)-\psi(a)\| .
$$

We can therefore conclude that:

$L_{m+1}\left(\psi, T_{j} ; a, b\right)$

$$
\begin{gathered}
\leqq \sum_{i \in \omega} \sum_{1 \leqq s \in d m n} \sum_{D_{i}} \sum_{r \in D_{i}^{*}(s)} \sum_{p=1}^{m+1}\left\{\left\|\psi\left(D_{i}(s-1)\right)-\psi\left(D_{i+1}(r-1)\right)\right\|^{m+1-p}\right. \\
\qquad 2 K\|\psi(b)-\psi(a)\| \cdot 2 K^{m}\|\psi(b)-\psi(a)\|^{m}=4 K^{1+m}\|\psi(b)-\psi(a)\|^{m+1} .
\end{gathered}
$$

4.5. TheOREM. Let $m$ be a positive integer, $\psi$ a function on $I$ to $E_{n+1}, S a$ subdivision of $I, K \geqq 1$, and for every $0 \leqq a \leqq b \leqq 1$ :

$$
L_{m}(\psi, S ; a, b) \leqq K^{m}\|\psi(b)-\psi(a)\|^{m} .
$$

Then

$$
\sum_{1 \leqq j \in d m n}\|\psi(S(j-1))-\psi(S(j))\|^{m} \leqq 2^{m+1} K^{2 m}\|\psi(1)-\psi(0)\|^{m} .
$$

Proof. Choose $D$ in $S(S ; 0,1)$ so that:

$$
\begin{aligned}
& \sum_{i \in \omega} \sum_{1 \leqq j \in \operatorname{dmn} D_{i}} \sum_{r} \in D_{D^{*}} \sum_{j} \sum_{p=1}^{m}\left\{\left\|\psi\left(D_{i}(j-1)\right)-\psi\left(D_{i+1}(r-1)\right)\right\|^{m-p}\right. \\
&\left.\cdot\left\|\psi\left(D_{i+1}(r-1)\right)-\psi\left(D_{i+1}(r)\right)\right\|^{p}\right\} \leqq K^{m}\|\psi(1)-\psi(0)\|^{m} .
\end{aligned}
$$

For $i \in \omega$ and $1 \leqq j \in \mathrm{dmn} D_{i}$, let 
4.5.1.

$$
d_{i}(j)=\inf \operatorname{Er}\left(D_{i+1}(r)>D_{i}(j-1)\right),
$$

4.5.2.

$$
\bar{D}_{i}(j)=D_{i+1}\left(d_{i}(j)\right) \text {. }
$$

We divide the remainder of the proof into six parts.

Part 1. If $D_{i}^{*}(j)=0$, then $\bar{D}_{i}(j)=D_{i}(j)$.

Proof. Compare definition 3.2.2 with 4.5.2.

Part 2. If $D^{\prime} \in \mathcal{S}\left(S ; D_{i}(j-1), D_{i}(j)\right)$, then

$$
D_{1}^{\prime}(1)=\bar{D}_{i}(j) \text {. }
$$

Proof. Observe that, in view of 3.2.3.4, if

$$
d_{i}{ }^{\prime}(j)=\inf \operatorname{Er}\left(S(r)>D_{i}(j-1)\right)
$$

we must have

$$
\bar{D}_{i}(j)=D_{i+1}\left(d_{i}(j)\right)=S\left(d_{i}{ }^{\prime}(j)\right)=D_{1}{ }^{\prime}(1) .
$$

Part 3. $\left\|\psi\left(D_{i}(j-1)\right)-\psi\left(\bar{D}_{i}(j)\right)\right\| \leqq 2 K\left\|\psi\left(D_{i}(j-1)\right)-\psi\left(D_{i}(j)\right)\right\|$ whenever $1 \leqq j \in \mathrm{dmn} D_{i}$.

Proof. By the hypothesis of the theorem, we have:

$$
L_{m}\left(\psi, S ; D_{i}(j-1), D_{i}(j)\right) \leqq K^{m}\left\|\psi\left(D_{i}(j-1)\right)-\psi\left(D_{i}(j)\right)\right\|^{m} .
$$

The desired conclusion then follows from part 2 and Lemma 4.3.

Part 4.

$$
\begin{aligned}
\sum_{1 \leqq j \in \operatorname{dmn}}\left\|\psi\left(D_{i+1}(j-1)\right)-\psi\left(\bar{D}_{i+1}(j)\right)\right\|^{m} & \\
= & \sum_{1 \leqq j \in \operatorname{dmn} D_{i}}\left\{\sum_{r \in D_{i^{*}(j)}}\left\|\psi\left(D_{i+1}(r-1)\right)-\psi\left(\bar{D}_{i+1}(r)\right)\right\|^{m}\right. \\
& \left.+\left\|\psi\left(D_{i}(j-1)\right)-\left(\bar{D}_{i}(j)\right)\right\|^{m}\right\} .
\end{aligned}
$$

Proof. Follows immediately from Lemma 4.2, and part 1.

Part 5. If $N \in \omega$, then

$$
\begin{aligned}
\sum_{1 \leqq i \in \operatorname{dmn} D_{N}}\left\|\psi\left(D_{N}(j-1)\right)-\psi\left(\bar{D}_{N}(j)\right)\right\|^{m} \\
=\sum_{i=0}^{N-1} \sum_{\substack{1 \leqq j \in \operatorname{dmn} D_{i} \\
r \in D_{i}^{*}(j)}}\left\|\psi\left(D_{i+1}(r-1)\right)-\psi\left(\bar{D}_{i+1}(r)\right)\right\|^{m} \\
\quad+\left\|\psi\left(D_{0}(0)\right)-\psi\left(\bar{D}_{0}(1)\right)\right\|^{m} .
\end{aligned}
$$

Proof. It is trivially true for $N=0$. By induction it must be true for all $N$ in $\omega$, in view of part 4.

Part 6. $\sum_{1 \leqq j \in d m n}\|\psi \psi(S(j-1))-\psi(S(j))\|^{m} \leqq 2^{m+1} K^{2 m}\|\psi(1)-\psi(0)\|^{m}$.

Proof. By 3.2.3.3, choose $N$ so that $D_{N}=S$. Then $D_{N+1}=D_{N}$ and hence $D_{N}^{*}(j)=0$ for all $1 \leqq j \in \mathrm{dmn} D_{N}$. Thus: 


$$
\begin{aligned}
\sum_{1 \leqq j \in d m n}\|\psi(S(j-1))-\psi(S(j))\|^{m} \\
=\left[\text { part 1] } \sum_{1 \leqq j \in d m n}\left\|\psi\left(D_{N}(j-1)\right)-\psi\left(\bar{D}_{N}(j)\right)\right\|^{m}\right. \\
=\left[\text { part 5] } \sum_{i=0}^{N-1} \sum_{1 \leqq j \in d m n} \sum_{r \in D_{i}^{*}(j)}\left\|\psi\left(D_{i+1}(r-1)\right)-\psi\left(\bar{D}_{i+1}(r)\right)\right\|^{m}\right. \\
\quad+\left\|\psi\left(D_{0}(0)\right)-\psi\left(\bar{D}_{0}(1)\right)\right\|^{m} \\
\leqq[\operatorname{part} 3] 2^{m} K^{m}\left\{\sum_{i=0}^{N-1} \sum_{1 \leqq j \in \operatorname{dmn} D_{i}} \sum_{r \in D_{i^{*}(j)}}\left\|\psi\left(D_{i+1}(r-1)\right)-\psi\left(D_{i+1}(r)\right)\right\|^{m}\right. \\
\leqq 2^{m} K^{m}\left\{K^{m}\|\psi(1)-\psi(0)\|^{m}+\|\psi(1)-\psi(0)\|^{m}\right\} \\
\leqq 2^{m+1} K^{2 m}\|\psi(1)-\psi(0)\|^{m} .
\end{aligned}
$$

4.6. CoRollary. If $\langle\langle m, \psi, T\rangle\rangle$, then there is a $K<\infty$ such that, if $0 \leqq a \leqq b$ $\leqq 1, i \in \omega, D$ is a subdivision of $[a, b]$ with $\operatorname{rng} D=\{a\} \cup\{b\} \cup\left(\operatorname{rng} T_{i}\right.$ $\cap[a, b])$, then

$$
\sum_{1 \leqq j \in \operatorname{dmn} D}\|\psi(D(j-1))-\psi(D(j))\|^{m} \leqq K\|\psi(b)-\psi(a)\|^{m} .
$$

4.7. Corollary. If $\langle\langle m, \psi, T\rangle\rangle$, then $\langle m, \psi, T\rangle$.

4.8. THEOREM. Let $\psi$ be a homeomorphism on $I$ to a subset of $E_{n+1}, T a$ mesh of $I$, and $\mathcal{L}$ denote Carathéodory linear measure. Then $\langle\langle 1, \psi, T\rangle\rangle$ if and only if there is a $K<\infty$ such that $\mathcal{L}(\psi[a, b]) \leqq K\|\psi(b)-\psi(a)\|$ whenever $0 \leqq a \leqq b \leqq 1$.

Proof. The sufficiency follows from the obvious fact that, if $S$ is a subdivision of $[a, b]$, then

$$
L_{1}(\psi, S ; a, b) \leqq \sum_{1 \leqq j \in \in_{\mathrm{dmn} S}}\|\psi(S(j-1))-\psi(S(j))\| \leqq \mathcal{L}(\psi[a, b]) .
$$

To show the necessity, let $\epsilon>0$ and choose $i \in \omega$ so that

$$
\mathcal{L}(\psi[0,1]) \leqq \sum_{1 \leqq s \in \operatorname{dmn} T_{i}}\left\|\psi\left(T_{i}(s-1)\right)-\psi\left(T_{i}(s)\right)\right\|+\epsilon .
$$

There exists a $K<\infty$ given by 4.6 such that, if $0 \leqq a \leqq b \leqq 1$ and $D$ is a subdivision of $[a, b]$ with $\operatorname{rng} D=\{a\} \cup\{b\} \cup\left(\mathrm{rng} T_{i} \cap[a, b]\right)$, then $\mathcal{L}(\psi[a, b]) \leqq \sum_{1 \leqq s \in d m n}\|\psi(D(s-1))-\psi(D(s))\|+\epsilon \leqq K\|\psi(b)-\psi(a)\|+\epsilon$.

Since $K$ is independent of the choice of $i$ and hence of $\epsilon$, we have the desired result. 
In $\S 6$ we shall need the following theorems.

4.9. ThEOREM. If $\psi$ is a linear map of $I$ into $E_{n+1}, S$ is any subdivision of $I, 0 \leqq a \leqq b \leqq 1$, then

$$
L_{2}(\psi, S ; a, b) \leqq\|\psi(b)-\psi(a)\|^{2} .
$$

4.10. Theorem. If $\psi$ is on $I$ to $E_{n+1}, S$ and $D$ are subdivisions of $[a, b]$, rng $D C$ rng $S$, and $m$ is a positive integer, then

$$
L_{m}(\psi, S ; a, b) \leqq L_{m}(\psi, D ; a, b)+\sum_{1 \leqq j \in \operatorname{dmn} D} L_{m}(\psi, S ; D(j-1), D(j)) .
$$

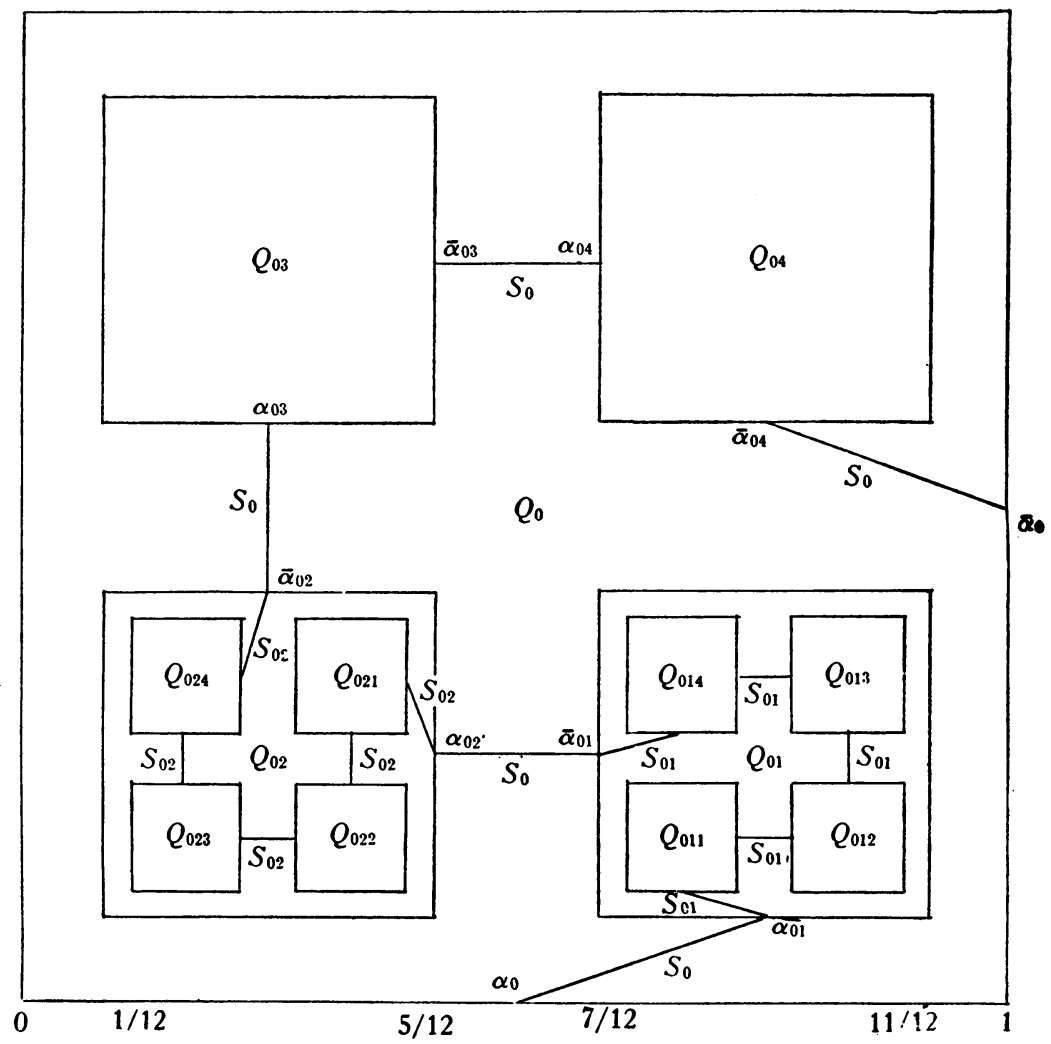

FIG. 1

5. Definition of Whitney's curve. We define Whitney's curve $P$ (see Whitney [4]) in detail only in the plane. In $\S 8$, we indicate how to construct it in $n$-dimensional space.

Referring to figure $1, Q_{0}$ is a unit square. It contains four squares of side $1 / 3$. Each of these contains in turn four squares of side $1 / 3^{2}$, and this process is continued indefinitely so that the side of every square is $1 / 3$ that of the 
smallest square containing it. The line segments $S_{0}, S_{01}, S_{02}$, etc. $\cdots$ join midpoints of the sides of the squares. The curve $P$ consists of these line segments and their limit points.

The remainder of this section is devoted to giving a detailed analytic definition of the curve $P$.

5.1. Given a square $A$, let $\mathrm{bm} A$, up $A$, lt $A, \mathrm{rt} A$ denote respectively the midpoint of the bottom side of $A$, the upper side of $A$, the left side of $A$, the right side of $A$.

5.2. If $x$ is the midpoint of a side of the square $A$, we shall denote by sm $x A B$ the midpoint of the same side of $B$ as that of $A$, i.e.:

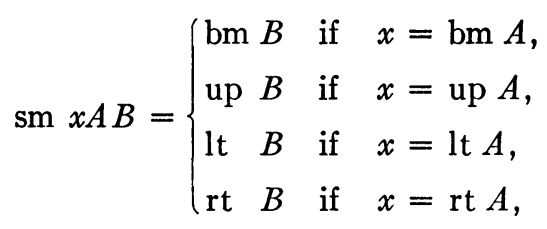

and op $x A B$ will stand for the midpoint of the opposite side of $B$ to that of $A$, i.e.:

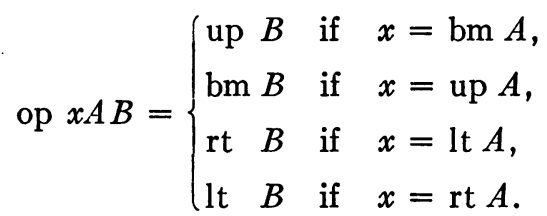

5.3. Given a square $A$, we define $\operatorname{rfnt} A$ to be the set of four disjoint squares each of side $1 / 3$ that of $A$ and each having its center coincide with the center of one of the four quadrants of $A$. Thus, the distance between two adjoining squares in $\operatorname{rfnt} A$ is $1 / 6$ the side of $A$, and the distance between a square in $\operatorname{rfnt} A$ and the boundary of $A$ is $1 / 12$ the side of $A$.

5.4. Given a square $A$ and the midpoints $a, b$ of two adjacent sides of $A$, we order the four squares in $\operatorname{rfnt} A$ in accordance with figure 1 , as follows: letting $a^{\prime}=$ op $a A A$ and $b^{\prime}=$ op $b A A$, we define the $j$ th square $Q^{*}(j ; A, a, b)$ by:

$$
Q^{*}(j ; A, a, b)=\text { The } B\left(B \in \operatorname { r f n t } A \text { and } \left\{\begin{array}{ll}
B \cap \operatorname{sgmt} a b \neq 0 & \text { if } j=1, \\
B \cap \operatorname{sgmt} a b^{\prime} \neq 0 & \text { if } j=2, \\
B \cap \operatorname{sgmt} a^{\prime} b^{\prime} \neq 0 & \text { if } j=3, \\
B \cap \operatorname{sgmt} a^{\prime} b \neq 0 & \text { if } j=4,
\end{array}\right.\right.
$$

and with it we associate the midpoints

$$
\alpha^{*}(j ; A, a, b), \bar{\alpha}^{*}(j ; A, a, b)
$$

of two of its adjacent sides, as follows: 


$$
\begin{array}{r}
\alpha^{*}(j ; A, a, b)=\left\{\begin{array}{lll}
\operatorname{sm} a A Q^{*}(j ; A, a, b) & \text { if } j=1, \\
\operatorname{sm} b A Q^{*}(j ; A, a, b) & \text { if } j=2, \\
\operatorname{sm} a A Q^{*}(j ; A, a, b) & \text { if } j=3, \\
\operatorname{op} b A Q^{*}(j ; A, a, b) & \text { if } j=4,
\end{array}\right. \\
\bar{\alpha}^{*}(j ; A, a, b)=\left\{\begin{array}{lll}
\operatorname{op} b A Q^{*}(j ; A, a, b) & \text { if } j=1, \\
\operatorname{op} a A Q^{*}(j ; A, a, b) & \text { if } j=2, \\
\operatorname{sm} b A Q^{*}(j ; A, a, b) & \text { if } j=3, \\
\operatorname{sm} a A Q^{*}(j ; A, a, b) & \text { if } j=4 .
\end{array}\right.
\end{array}
$$

For convenience later on, we also let

$$
\bar{\alpha}^{*}(0 ; A, a, b)=a \text { and } \quad \alpha^{*}(5 ; A, a, b)=b .
$$

5.5. The line segments, being part of the curve $P$, will be defined by recursion. For this reason, we introduce the following definitions.

5.5.1. For every $n \in \omega$, let $H_{n}$ be the set of sequences $k$ of integers such that $k_{0}=0,1 \leqq k_{j} \leqq 4$ whenever $1 \leqq j \leqq n, k_{j}=0$ for $j>n$.

5.5.2. $H=\bigcup_{n \in \omega} H_{n}$.

5.5.3. For every $k$ in $H$, let $\operatorname{dim} k$ be the $n \in \omega$ such that $k \in H_{n}$.

5.5.4. We shall denote by $\theta$ the $k$ in $H_{0}$; thus, $\theta_{j}=0$ for all $j \in \omega$.

5.5.5. If $k$ is in $H_{n}$ and $n>0$, we denote by $k^{*}$ the sequence in $H_{n-1}$ such that $k_{j}^{*}=k_{j}$ whenever $0 \leqq j \leqq n-1$.

5.5.6. For every $k$ in $H$, let $\bar{S}(k)$ be the set of sequences $q$ in $H$ such that $q_{j}=k_{j}$ whenever $0 \leqq j \leqq \operatorname{dim} k$.

5.5.7. If $k$ is in $H_{n}$ and $j$ is an integer with $1 \leqq j \leqq 4$, we denote by $s(j, k)$ the $q$ in $\bar{S}(k) \cap H_{n+1}$ such that $q_{n}=j$.

5.6. With each $k$ in $H$ we associate a square $Q_{k}$ and midpoints $\alpha_{k}, \bar{\alpha}_{k}$ of two of its adjacent sides. This is done by recursion on $\operatorname{dim} k$.

We let $Q_{\theta}$ be a unit square, $\alpha_{\theta}=\mathrm{bm} Q_{\theta}$, and $\bar{\alpha}_{\theta}=\mathrm{rt} Q_{\theta}$. If $n \in \omega$ and $\operatorname{dim} k$ $=n+1$, then we let

$$
\begin{aligned}
& Q_{k}=Q^{*}\left(k_{n} ; Q_{k^{*}}, \alpha_{k^{*}}, \bar{\alpha}_{k^{*}}\right), \\
& \alpha_{k}=\alpha^{*}\left(k_{n} ; Q_{k^{*}}, \alpha_{k^{*}}, \bar{\alpha}_{k^{*}}\right), \\
& \bar{\alpha}_{k}=\bar{\alpha}^{*}\left(k_{n} ; Q_{k^{*}}, \alpha_{k^{*}}, \bar{\alpha}_{k^{*}}\right) .
\end{aligned}
$$

The following abbreviations are introduced for convenience.

5.6.1. $Q^{\prime}(j, k)=Q^{*}\left(j ; Q_{k}, \alpha_{k}, \bar{\alpha}_{k}\right), j=1, \cdots, 4$.

5.6.2. $\alpha^{\prime}(j, k)=\alpha^{*}\left(j+1 ; Q_{k}, \alpha_{k}, \bar{\alpha}_{k}\right), j=0, \cdots, 4$.

5.6.3. $\bar{\alpha}^{\prime}(j, k)=\bar{\alpha}^{*}\left(j ; Q_{k}, \alpha_{k}, \bar{\alpha}_{k}\right), j=0, \cdots, 4$.

5.7. We define the five line segments in $Q_{k}$ for $k$ in $H$ by:

5.7.1. line $j k=\operatorname{sgmt} \bar{\alpha}^{\prime}(j, k) \alpha^{\prime}(j, k), j=0, \cdots, 4$, and we define their union by: 


$$
S_{k}=\bigcup_{j=0}^{4} \text { line } j k
$$

5.8. Denoting the set of line segments by $P^{\prime}$, i.e.:

we define the curve $P$ by:

$$
P^{\prime}=\bigcup_{k \in H} S_{k}
$$

5.8.2. $P=$ closure of $P^{\prime}$.

6. Some properties of the curve $P$. The next three lemmas are immediate consequences of the definitions.

6.1. Lemma. If $k \in H_{n}$, then the side of $Q_{k}$ has length $1 / 3^{n}$ and hence diameter $Q_{k} \leqq 2 / 3^{n}$.

6.2. Lemma. If $k \in H, q \in \bar{S}(k)$, then $Q_{q} \subset Q_{k}$.

6.3. Lemma. If $k \in H, j=1, \cdots, 4$, then $Q^{\prime}(j, k)=Q_{s(j, k)}, \alpha^{\prime}(j-1, k)$ $=\alpha_{s(j, k)}$, and $\bar{\alpha}^{\prime}(j, k)=\bar{\alpha}_{s(j, k)}$.

6.4. Theorem. If $x, y, a$ are such that sgmt $x y$ is not in $P$, sgmt $x a \in P$ and sgmt $y a \subset P$, then

$$
\|y-a\| \leqq 10^{1 / 2}\|x-y\| \text { and }\|x-a\| \leqq 10^{1 / 2}\|x-y\| .
$$

Proof. If $(\gamma / 2)$ is the angle between sgmt $x a$ and sgmt $y a$, then tan $\gamma>1 / 3$ and $\sin (\gamma / 2)>1 / 10^{1 / 2}$.

6.5. THEOREM. If $x$ and $y$ are in $P, k \in H_{n}, Q_{k}$ is the smallest square containing both $x$ and $y$, and there is no $a$ such that sgmt $x a \subset P$ and sgmt $y a \subset P$, then $\|x-y\| \geqq(1 / 12) 1 / 3^{n+1}$.

Proof. If $x$ and $y$ are in $S_{k}$, then they must be on two different line segments and hence $\|x-y\| \geqq(1 / 6) 1 / 3^{n}$. If $x$ is in $S_{k}$ and $y$ is in $Q^{\prime}(j, k)$ for some $j=1, \cdots, 4$, then $\|x-y\| \geqq(1 / 12) 1 / 3^{n+1}$. If $x$ is in $Q^{\prime}(j, k)$ and $y$ is in $Q^{\prime}\left(j^{\prime}, k\right)$, then $j \neq j^{\prime}$ and $\|x-y\| \geqq(1 / 6) 1 / 3^{n}$.

In order to show that $P$ is a homeomorphic image of the unit interval, we introduce the following definitions.

6.6. Definition. For $k$ in $H_{n}$, we define:

$$
\begin{aligned}
a_{k} & =\sum_{p=1}^{n}\left(2 k_{p}-1\right) / 9^{p}, & & \\
\bar{a}_{k} & =a_{k}+1 / 9^{n}, & & \\
a^{\prime}(j, k) & =a_{k}+(2 j+1) / 9^{n+1} & & \text { for } j=0, \cdots, 4, \\
\bar{a}^{\prime}(j, k) & =a_{k}+(2 j) / 9^{n+1} & & \text { for } j=0, \cdots, 4 .
\end{aligned}
$$

Notice that all these points are in $I$, that: 


$$
\begin{array}{rlrl}
a_{k} & \leqq \bar{a}^{\prime}(j, k) \leqq a^{\prime}(j, k) \leqq \bar{a}_{k} & \text { for } j=0, \cdots, 4, \\
a_{k} & =\bar{a}^{\prime}(0, k), \quad \bar{a}_{k}=a^{\prime}(4, k), & \\
a^{\prime}(j, k) & =a_{s(j+1, k),} \quad \bar{a}^{\prime}(j+1, k)=\bar{a}_{s(j+1, k)} & & (j=0, \cdots, 3) .
\end{array}
$$

6.7. Definition. We define the mapping $\psi$ on $I$ to $P$ to be such that for $k$ in $H_{n}$ and $j=0, \cdots, 4, \psi$ maps the interval $\left[\bar{a}^{\prime}(j, k), a^{\prime}(j, k)\right]$ linearly onto line $j k$ with $\psi\left(\bar{a}^{\prime}(j, k)\right)=\bar{\alpha}^{\prime}(j, k)$ and $\psi\left(a^{\prime}(j, k)\right)=\alpha^{\prime}(j, k)$. It is then extended to the whole interval $I$ by continuity.

Thus, $\psi$ maps the interval $\left[a_{k}, \bar{a}_{k}\right]$ on to $P \cap Q_{k}$ for $k$ in $H$. It is obviously a homeomorphism on $I$ to $P$.

6.8. Definitions.

6.8.1. Let $T$ be the mesh of $I$ such that, for every $i \in \omega, \operatorname{dmn} T_{i}$ $=\mathrm{E} s\left(s=0, \cdots, 9^{i}\right)$ and, for $s$ in $\operatorname{dmn} T_{i}, T_{i}(s)=s / 9^{i}$.

6.8.2. $A_{i}\left(t, t^{\prime}\right)=\mathrm{E} s\left(t \leqq T_{i}(s-1)<T_{i}(s) \leqq t^{\prime}\right)$.

6.8.3. $\bar{t}_{i}=\inf \left(\operatorname{rng} T_{i} \cap[t, 1]\right)$.

6.8.4. $t_{i}^{*}=\sup \left(\mathrm{rng} T_{i} \cap[0, t]\right)$.

REMARK. Notice that if $t \in \mathrm{rng} T_{i}$, then $t=\bar{t}_{i}=t_{i}^{*}$.

The remainder of this section is devoted to proving $\langle\langle 2, \psi, T\rangle\rangle$.

6.9. Lemma. If $k \in H_{n}$, then

$$
\begin{aligned}
\operatorname{rng} T_{n+1} \cap\left[a_{k}, \bar{a}_{k}\right] & =\bigcup_{j=0}^{4}\left(\left\{a^{\prime}(j, k)\right\} \cup\left\{\bar{a}^{\prime}(j, k)\right\}\right), & \\
\operatorname{rng} T_{n} \cap\left[a_{k}, \bar{a}_{k}\right] & =\left\{a_{k}\right\} \cup\left\{\bar{a}_{k}\right\}, & \\
\operatorname{rng} T_{i} \cap\left[a_{k}, \bar{a}_{k}\right] & =0 & \text { for } i<n .
\end{aligned}
$$

6.10. Lemma. If $i \in \omega, k \in H_{n}, j=0, \cdots, 4, \bar{a}^{\prime}(j, k) \leqq t \leqq t^{\prime} \leqq a^{\prime}(j, k)$, then

$$
L_{2}\left(\psi, T_{i} ; t, t^{\prime}\right) \leqq\left\|\psi\left(t^{\prime}\right)-\psi(t)\right\|^{2} \leqq 4 / 3^{2 n}
$$

Proof. $\psi\left[\bar{a}^{\prime}(j, k), a^{\prime}(j, k)\right]=$ line $j k \subset Q_{k}$. Hence, by 4.9 and 6.1,

$$
L_{2}\left(\psi, T_{i} ; t, t^{\prime}\right) \leqq\left\|\psi\left(t^{\prime}\right)-\psi(t)\right\|^{2} \leqq\left(\text { diameter } Q_{k}\right)^{2} \leqq 4 / 3^{2 n} .
$$

6.11. LEMmA. If $k \in H_{n}, a_{k} \leqq t \leqq t^{\prime} \leqq \bar{a}_{k}$, then

$$
L_{2}\left(\psi, T_{n+1} ; t, t^{\prime}\right) \leqq 64 / 3^{2 n} \text {. }
$$

Proof.

$$
\begin{aligned}
L_{2}\left(\psi, T_{n+1} ; t, t^{\prime}\right) \leqq & \sum_{\bullet \in A n+1} \sum_{p=1}^{2}\left\{\left\|\psi(t)-\psi\left(T_{n+1}(s-1)\right)\right\|^{2-p}\right. \\
\left.\cdot\left\|\psi\left(T_{n+1}(s-1)\right)-\psi\left(T_{n+1}(s)\right)\right\|^{p}\right\} & \\
& +\sum_{p=1}^{2}\left\{\left\|\psi(t)-\psi\left(t_{n+1}^{\prime *}\right)\right\|^{2-p}\left\|\psi\left(t_{n+1}^{\prime *}\right)-\psi\left(t^{\prime}\right)\right\| p\right\} \\
\leqq & {[6.9] 8 \cdot 2\left(\operatorname{diameter} Q_{k}\right)^{2} \leqq[6.1] 16\left(4 / 3^{2 n}\right)=64 / 3^{2 n} . }
\end{aligned}
$$


6.12. Lemma. If $k \in H_{n}, i \in \omega$, then

$$
\begin{aligned}
L_{2}\left(\psi, T_{i} ; a_{k}, \bar{a}_{k}\right) \leqq & L_{2}\left(\psi, T_{n+1} ; a_{k}, \bar{a}_{k}\right)+\sum_{j=0}^{4} L_{2}\left(\psi, T_{i} ; \bar{a}^{\prime}(j, k), a^{\prime}(j, k)\right) \\
& +\sum_{j=0}^{3} L_{2}\left(\psi, T_{i} ; a^{\prime}(j, k), \bar{a}^{\prime}(j+1, k)\right) .
\end{aligned}
$$

Proof. The proof follows immediately from 4.10 and 6.9.

6.13. Lemma. If $k \in H_{n}$ and $i \in \omega$, then

$$
\begin{aligned}
& L_{2}\left(\psi, T_{i} ; a_{k}, \bar{a}_{k}\right) \leqq \sum_{s=0}^{i-n-1} \sum_{k^{\prime} \in \bar{s}(k) H_{n+s}}\left\{L_{2}\left(\psi, T_{n+s+1} ; a_{k^{\prime}}, \bar{a}_{k^{\prime}}\right)\right. \\
& \left.+\sum_{j=0}^{4} L_{2}\left(\psi, T_{i} ; \bar{a}^{\prime}\left(j, k^{\prime}\right), a^{\prime}\left(j, k^{\prime}\right)\right)\right\} .
\end{aligned}
$$

Proof. In view of the remarks at the end of 6.6 and Definition 5.5.7, we have:

$$
\begin{aligned}
& \sum_{j=0}^{8} L_{2}\left(\psi, T_{i} ; a^{\prime}(j, k), \bar{a}^{\prime}(j+1, k)\right) \\
& =\sum_{k^{\prime} \in \bar{S}(k) \cap B_{n+1}} L_{2}\left(\psi, T_{i} ; a_{k^{\prime}}, \bar{a}_{k^{\prime}}\right) \\
& \leqq[6.12] \sum_{k^{\prime} \in \bar{S}(k) \cap B_{n+1}}\left\{L_{2}\left(\psi, T_{n+2} ; a_{k^{\prime}}, \bar{a}_{k^{\prime}}\right)\right. \\
& \left.+\sum_{j=0}^{4} L_{2}\left(\psi, T_{i} ; \bar{a}^{\prime}\left(j, k^{\prime}\right), a^{\prime}\left(j, k^{\prime}\right)\right)+\sum_{k^{\prime \prime} \in \bar{s}\left(k^{\prime}\right) \cap_{H_{n+2}}} L_{2}\left(\psi, T_{i} ; a_{k^{\prime \prime}}, \bar{a}_{k^{\prime \prime}}\right)\right\} \\
& =\sum_{k^{\prime} \in \bar{s}(k) \cap_{H_{n+1}}}\left\{L_{2}\left(\psi, T_{n+2} ; a_{k^{\prime}}, \bar{a}_{k^{\prime}}\right)+\sum_{j=0}^{4} L_{2}\left(\psi, T_{i} ; \bar{a}^{\prime}\left(j, k^{\prime}\right), a^{\prime}\left(j, k^{\prime}\right)\right)\right\} \\
& +\sum_{k^{\prime \prime} \in \bar{s}(k) \cap H_{n+2}} L_{2}\left(\psi, T_{i} ; a_{k^{\prime \prime}}, \bar{a}_{k^{\prime \prime}}\right) \text {. }
\end{aligned}
$$

Applying 6.12, induction on $i-n$, and 6.9, we have the desired result.

6.14. THEOREM. If $k \in H_{n}$ and $i \in \omega$, then

$$
L_{2}\left(\psi, T_{i} ; a_{k}, \bar{a}_{k}\right) \leqq 168 / 3^{2 n} \text {. }
$$

Proof. Using 6.13, 6.11, and 6.10 we see that:

$$
\begin{aligned}
L_{2}\left(\psi, T_{i} ; a_{k}, \bar{a}_{k}\right) & \leqq \sum_{s=0}^{i-n-1} \sum_{k^{\prime} \in \bar{s}(k) n_{H_{n+s}}\left\{64 / 3^{2(n+s)}+5\left(4 / 3^{2(n+s)}\right)\right\}} \\
& =\sum_{s=0}^{i-n-1} 4^{s}\left(84 / 3^{2(n+s)}\right) \leqq\left(84 / 3^{2 n}\right) \sum_{s \in \omega}(4 / 9)^{*}<168 / 3^{2 n} .
\end{aligned}
$$


6.15. Lemma. If $k \in H_{n}, a_{k} \leqq t \leqq t^{\prime} \leqq \bar{a}_{k}, i \in \omega$, then

$$
L_{2}\left(\psi, T_{i} ; t, t^{\prime}\right) \leqq 252 / 3^{2 n}+L_{2}\left(\psi, T_{i} ; t, \bar{t}_{n+1}\right)+L_{2}\left(\psi, T_{i} ; t_{n+1}^{\prime *}, t^{\prime}\right) .
$$

\section{Proof.}

$$
\begin{aligned}
L_{2}\left(\psi, T_{i} ; t, t^{\prime}\right) \leqq & {[4.10] L_{2}\left(\psi, T_{n+1} ; t, t^{\prime}\right)+L_{2}\left(\psi, T_{i} ; t, \bar{t}_{n+1}\right) } \\
& +L_{2}\left(\psi, T_{i} ; t_{n+1}^{\prime *}, t^{\prime}\right)+\sum_{j \in A_{n+1}\left(t, t^{\prime}\right)} L_{2}\left(\psi, T_{i} ; T_{n+1}(j-1), T_{n+1}(j)\right) \\
\leqq & {[6.11,6.9,6.10,6.14] 64 / 3^{2 n}+L_{2}\left(\psi, T_{i} ; t, \bar{t}_{n+1}\right) } \\
& +L_{2}\left(\psi, T_{i} ; t_{n+1}^{\prime *}, t^{\prime}\right)+5\left(4 / 3^{2 n}\right)+4\left(168 / 3^{2 n+2}\right) \\
\leqq & 252 / 3^{2 n}+L_{2}\left(\psi, T_{i} ; t, \bar{t}_{n+1}\right)+L_{2}\left(\psi, T_{i} ; t_{n+1}^{\prime *}, t^{\prime}\right) .
\end{aligned}
$$

6.16. Lemma. If $k \in H_{n}, a_{k} \leqq t \leqq t^{\prime} \leqq \bar{a}_{k}, i \in \omega$, and $1 \leqq j \in \omega$, then

$$
L_{2}\left(\psi, T_{i} ; t, t^{\prime}\right) \leqq 1008 / 3^{2 n}+L_{2}\left(\psi, T_{i} ; t, \bar{t}_{n+j}\right)+L_{2}\left(\psi, T_{i} ; t_{n+j}^{\prime *}, t^{\prime}\right) .
$$

Proof. The desired conclusion will follow if we show

$$
L_{2}\left(\psi, T_{i} ; t, t^{\prime}\right) \leqq 2 \sum_{s=1}^{i} 252 / 3^{2(n+8-1)}+L_{2}\left(\psi, T_{i} ; t, \bar{t}_{n+j}\right)+L_{2}\left(\psi, T_{i} ; t_{n+j}^{\prime *}, t^{\prime}\right) .
$$

We prove the above inequality by induction. By 6.15 it holds for $j=1$. Now suppose it holds for $j=r, 1 \leqq r \in \omega$.

Notice first that, if $c=\bar{t}_{n+r}$ and $d=t_{n+r}^{\prime *}$, then $c$ and $d$ are in rng $T_{n+r}$ and hence, by the remark at the end of $6.8, c_{n+r+1}^{*}=c$ and $\bar{d}_{n+r+1}=d$ so that $L_{2}\left(\psi, T_{i}\right.$; $\left.c_{n+r+1}^{*}, c\right)=L_{2}\left(\psi, T_{i} ; d, \bar{d}_{n+r+1}\right)=0$. Thus, if for some $k^{\prime}$ and $k^{\prime \prime}$ in $H_{n+r}$ we have $a_{k^{\prime}} \leqq t \leqq \bar{a}_{k^{\prime}}$ and $a_{k^{\prime \prime}} \leqq t^{\prime} \leqq \bar{a}_{k^{\prime \prime}}$, then by 6.15

$$
\begin{aligned}
& L_{2}\left(\psi, T_{i} ; t, \bar{t}_{n+r}\right) \leqq 252 / 3^{2(n+r)}+L_{2}\left(\psi, T_{i} ; t, \bar{t}_{n+r+1}\right), \\
& L_{2}\left(\psi, T_{i} ; t_{n+r}^{\prime *}, t^{\prime}\right) \leqq 252 / 3^{2(n+r)}+L_{2}\left(\psi, T_{i} ; t_{n+r+1}^{\prime *}, t^{\prime}\right),
\end{aligned}
$$

and the desired conclusion for $j=r+1$ follows.

If for no $k^{\prime}$ in $H_{n+r}$ do we have $a_{k^{\prime}} \leqq t \leqq \bar{a}_{k^{\prime}}$, then for some $r^{\prime} \leqq r$ and some $q$ in $H_{n+r^{\prime}}$, we must have $\bar{a}_{q} \leqq t \leqq a_{q}$, and hence by 6.10 :

$$
L_{2}\left(\psi, T_{i} ; t, \bar{t}_{n+r}\right) \leqq\left\|\psi(t)-\psi\left(\bar{t}_{n+r}\right)\right\|^{2} \leqq 4 / 3^{2(n+r)} .
$$

Similarly, if for no $k^{\prime}$ in $H_{n+r}$ do we have $a_{k^{\prime}} \leqq t^{\prime} \leqq \bar{a}_{k^{\prime}}$, then

$$
L_{2}\left(\psi, T_{i} ; t_{n+r}^{*}, t^{\prime}\right) \leqq 4 / 3^{2(n+r)}
$$

and we have the desired result.

6.17. ThEOREM. If $k \in H_{n}, a_{k} \leqq t \leqq t^{\prime} \leqq \bar{a}_{k}$, and $i \in \omega$, then 


$$
L_{2}\left(\psi, T_{i} ; t, t^{\prime}\right) \leqq 1008 / 3^{2 n} .
$$

Proof. Pick $j \in \omega$ so that $i<n+j$. Then

$$
L_{2}\left(\psi, T_{i} ; t, \bar{t}_{n+j}\right)=L_{2}\left(\psi, T_{i} ; t_{n+j}^{\prime *}, t^{\prime}\right)=0
$$

and the conclusion follows from 6.16 .

6.18. Theorem. $\langle\langle 2, \psi, T\rangle\rangle$.

Proof. Let $0 \leqq t \leqq t^{\prime} \leqq 1, x=\psi(t), y=\psi\left(t^{\prime}\right)$. If sgmt $x y \subset P$, then, by 4.9 , $L_{2}\left(\psi, T_{i} ; t, t^{\prime}\right) \leqq\|y-x\|^{2}$.

If sgmt $x y$ is not in $P$, but, for some $a$, sgmt $x a \subset P$ and sgmt $y a \subset P$, then

$$
\begin{aligned}
L_{2}\left(\psi, T_{i} ; t, t^{\prime}\right) \leqq & {[4.10]\|x-a\| \cdot\|y-a\|+\|y-a\|^{2} } \\
& +L_{2}\left(\psi, T_{i} ; t, \psi^{-1}(a)\right)+L_{2}\left(\psi, T_{i} ; \psi^{-1}(a), t^{\prime}\right) \\
\leqq & {[4.9]\|x-a\| \cdot\|y-a\|+\|y-a\|^{2}+\|x-a\|^{2} } \\
& +\|y-a\|^{2} \leqq[6.4] 40\|x-y\|^{2} .
\end{aligned}
$$

If $k$ is in $H_{n}$ and $Q_{k}$ is the smallest square containing both $x$ and $y$ and there is no $a$ such that sgmt $x a \subset P$ and sgmt $y a \subset P$, then by 6.17 and 6.5 we have

$$
L_{2}\left(\psi, T_{i} ; t, t^{\prime}\right) \leqq 1008 / 3^{2 n}=1008 \cdot 3^{2} \cdot 12^{2} /\left(12^{2} \cdot 3^{2 n+2}\right) \leqq 1008 \cdot 3^{2} \cdot 12^{2}\|x-y\|^{2} .
$$

7. Functions not constant on a connected set of zeros of their higher order partials. H. Whitney [4] has shown the existence of a function $W$ on the curve $P$ of $\S 5$ such that, if $R^{\prime}(x, y)=W(y)-W(x)$, then $R^{\prime}$ is a remainder of order 1 on $(P \times P)$, and yet $W$ assumes the values 0 and 1 respectively at two different points of $P$.

7.1. TheOREM. There exists a function $g$ of class $C^{2}$ on $E_{2}$ such that: all of its second order partials vanish everywhere on $P$; all of its first order partials vanish at one point of $P$; and yet $g$ is not constant on $P$.

Proof. Let $\psi$ and $T$ be respectively the homeomorphism and mesh defined in 6.7 and 6.8. Then $P=\psi(I)$ and, by $6.18,\langle\langle 2, \psi, T\rangle\rangle$. Let $k^{\prime}$ be the $k$ in $O_{2}$ such that $k_{0}=1$ and $k_{j}=0$ for $1 \leqq j \in \omega$. Let $F$ and $R$ be functions on $O_{2}$ such that $F_{k^{\prime}}=W, R_{k^{\prime}}=R^{\prime}$, and $F_{k}=R_{k}=0$ for all other $k$ in $O_{2}$ ( $W$ and $R^{\prime}$ being Whitney's function and remainder stated above). Then, we see immediately that we have $[2, F, R, \psi(I)]$ and hence by 4.7 and 3.3.19 we have the desired result.

7.2. TheOREM. For any integer $m \geqq 1$ there exists a function of class $C^{m}$ on $E_{2}$ such that: all of its partials of order $m$ vanish everywhere on $P$; all of its partials vanish at one point of $P$; the function is not constant on $P$. 
Proof. The proof is by induction on $m$, using 4.4 and the method of 7.1.

8. Extensions to higher dimensions. In $E_{n}, 2 \leqq n \in \omega$, Whitney's curve is constructed by starting with an $n$-cell of side 1 , and then taking $2^{n}$ cells inside, each of side $r$ where $r<1 / 2$ and $r^{n-1}>1 / 2^{n}$. Inside each of these, we again take $2^{n}$ cells each of side $r^{2}$. This process is continued indefinitely so that inside each cell we take $2^{n}$ cells of side $r$ times that of its own. Then, as before, the curve $P$ will consist of line segments, joining midpoints of appropriate faces of these cells, and their limit points. As in the case of $n=2$, it can be seen that there is a homeomorphism $\psi$ and a mesh $T$ of $I$ such that $\psi(I)=P$ and $\langle\langle n, \psi, T\rangle\rangle$. The choice of $r$ enables Whitney to define a function $W$, not constant on $P$, such that, if $R(x, y)=W(y)-W(x)$, then $R$ is a remainder of order $(n-1)$ with domain $(P \times P)$. We may then use Theorem 3.3.19 and induction as before to prove the

8.1. Theorem. If $m \in \omega$ and $2 \leqq n \in \omega$, there exists a homeomorphic image $P$ of the unit interval and a function $g$ of class $C^{(n+m-1)}$ on $E_{n}$ such that: all of its partials of order greater than $m$ vanish everywhere on $P$; all of its partials vanish at one point of $P$; and $g$ is not constant on $P$.

8.2. Remark. In view of A. P. Morse's result, $P$ must have infinite Carathéodory $(n-1)$-measure, and yet we have $\langle\langle n, \psi, T\rangle\rangle, \psi$ and $T$ being taken as above.

\section{BIBLIOGRAPHY}

1. A. P. Morse, The behavior of a function on its critical set, Ann. of Math. vol. 40 (1939) pp. $62-70$.

2. A. Sard, The measure of the critical values of differentiable maps, Bull. Amer. Math. Soc. vol. 48 (1942) pp. 883-890.

3. H. Whitney, Analytic extensions of differentiable functions defined on closed sets, Trans. Amer. Math. Soc. vol. 36 (1934) pp. 63-89.

4. - A function not constant on a connected set of critical points, Duke Math. J. vol. 1 (1935) pp. 514-517.

5. W. M. Whyburn, Non-isolated critical points of functions, Bull. Amer. Math. Soc. vol. 35 (1929) pp. 701-708.

University OF CALIFornia, Berkeley, Calif. 